\title{
Wie Kinder Essen erleben und erlernen
}

\section{Edith Gätjen}

\author{
Erwachsene haben Erwartungen... Kinder sollen erst flüssig, dann breiig, zuletzt stückig, \\ stets planmäßig, möglichst sauber, immer in der vorgesehenen Menge, besonders \\ gesund, vollwertig, durchgehend freudig, gerne neugierig ernährt werden! Aber gut \\ essende Kinder fallen nicht einfach von den Bäumen. Denn Kinder haben Bedürfnisse; sie \\ wollen in Beziehung, mit Begleitung, Selbstvertrauen, Verlässlichkeit, Kontinuität und mit \\ Aufmerksamkeit essen lernen.
}

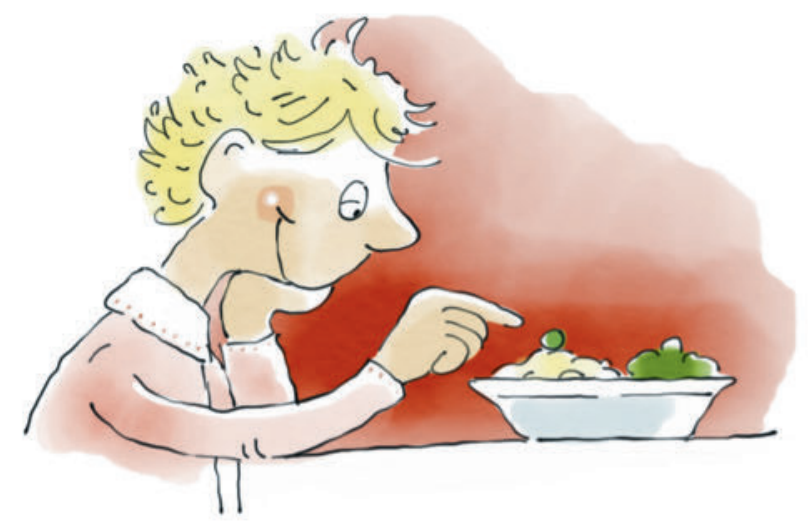

Abb. 1 Mit den Lebensmitteln flirten: Das ist ein natürliches Verhalten der kindlichen Essentwicklung. Quelle: Verlag Eugen Ulmer, Stuttgart, mit freundlicher Genehmigung

Wie ist die Situation? Am Tisch sitzen sich das „Sollen“ und das „Wollen“ gegenüber und nicht selten behindert das „Sollen“ die Wahrnehmung des „Wollens“. Je größer die Diskrepanz zwischen den Erwartungen der Erwachsenen und den Bedürfnissen der Kinder ist, umso schwieriger gestaltet sich die Situation am Esstisch und somit die Entwicklung eines selbstbestimmten, gesundheitsförderlichen und genussvollen Essverhaltens. Damit die Erwartungen der Erwachsenen, das „Sollen“, und die Bedürfnisse der Kinder, das „Wollen“, sich annähern können, braucht es das Wissen über die Entwicklung des kindlichen Essverhaltens und die Bedeutung einer Mahlzeit sowie die Einflussfaktoren auf das kindliche Essverhalten ( $\triangleright$ Abb. 1).

\section{Merke}

Das Essverhalten wird bestimmt durch biologische und physiologische Vorgänge, Geschmacksentwicklung und kulturelle Prägung, Evolution und Genetik sowie Beziehung, Erfahrungen und Erziehung.

\section{Phasen der Essentwicklung}

Die Ernährung und das Essverhalten von Anfang an bis zum Erwachsenenalter kann man grob in 6 Phasen einteilen. Diese Phasen sind geprägt von der Entwicklung des Verdauungstraktes, der Motorik, der physischen und psychischen Bedürfnisse sowie den Entwicklungsaufgaben eines Kindes in dem Spannungsfeld „Bindung - Ablösung - Autonomie“. Bezeichnend für diese Phasen ist die Art und Weise, wie gegessen wird, was, wann und warum sowie das dahinterliegende Motiv.

In der ersten Phase im Mutterleib handelt es sich um eine passive, kontinuierliche Nahrungszufuhr niedermolekularer Nährstoffe und Antikörper über die Nabelschnur - dies 24 Stunden am Tag - und es dient dem körperlichen Aufbau des Kindes.

Die zweite Phase beginnt mit der Geburt, auch der Hunger wird geboren und der Säugling saugt aktiv Milch, 
gesteuert über Reflexe, liegend und liebevoll gehalten. Diese hat Körpertemperatur und ist angepasst an das noch unreife Verdauungssystem. Hier wird in ein- und demselben Prozess der physiologische (Kalorien und Nährstoffe) und emotionale (Beziehung, Lust, Orientierung, Selbstwerterhöhung) Hunger gesättigt. Das Angebot bestimmen die Eltern, der Säugling bedient sich, bis zu ca. 16-mal in 24 Stunden, seine Bedürfnisse werden sofort befriedigt.

Das zweite Lebenshalbjahr beschreibt die dritte Phase. Der Säugling saugt weiterhin Milch, hinzu kommt das Essen von halbfester Nahrung und das Trinken aus dem Glas. Essen wird gelernt, liebevoll gehalten, sitzend. Außer Milch gibt es Mus und Brei, warm oder kalt sowie Weiches und bröseliges Fingerfood. Angebot und Menge bestimmen die Eltern, Tagespflege, Erzieher, das Kind nimmt sich - ca. 6-mal in 24 Stunden. Neugierde kommt als Motiv hinzu.

Ab dem ersten Geburtstag beginnt die vierte Phase und der langsame Abschied von dem Saugen und zunehmendem Essen von stückiger, fester Kost mit den Eltern von einem gemeinsamen Teller, selbstständig sitzend auf dem Schoß der Eltern. Angepasst an die Zahnentwicklung gibt es ein abwechslungsreiches Angebot. Das Essen ist mehr und mehr an Mahlzeiten gebunden, ca. 4-5 pro Tag, und die direkte Bedürfnisbefriedigung nimmt ab. Zur Neugierde kommt das Imitieren und der Spaß am Essen und der Gemeinschaft.

Die fünfte Phase beginnt mit $11 / 2$ Jahren, selbstständiges Portionieren und selbstständiges Essen mit Besteck gelingt. Das Kind sitzt mit am Tisch. Alle essen bei Familienmahlzeiten und Kita-Mahlzeiten das Gleiche (unter Berücksichtigung von Unverträglichkeiten und Allergien). Verschiedene Konsistenzen und Temperaturen werden angeboten, das Kind bedient sich, Erwachsene und andere Kinder sind „Vorleber“. Vier bis fünf gemeinsame Mahlzeiten, der Bedürfnisaufschub wird gelernt. Gegessen und getrunken wird aus Hunger und Durst, aber auch Appetit, Motive wie Beschäftigung, Gemeinschaftserleben, Essensneid und Trotz haben einen Einfluss auf das Essverhalten. Der physiologische Hunger wird gesättigt über die Lebensmittel, Kalorien und Nährstoffe, der emotionale Hunger wird gesättigt über Kontakt und Kommunikation bei gemeinsamen Mahlzeiten.

Zum Ende der Grundschulzeit beginnt die sechste Phase, hier werden Mahlzeiten selbstständig organisiert und gestaltet; es wird alleine gegessen, mit Freunden, in der Schule, unterwegs und in der Familie. Motive wie „Trends“, „Image“, „Schönsein“, „sportliche Leistung“, „Ökologisches“, „Politik/Soziales“, „Abgrenzung von den Eltern“ spielen eine große Rolle.

\section{PRAXISTIPP}

Zwischen den 6 Phasen der Entwicklung des kindlichen Essverhaltens sind in der Regel fließende Übergänge - außer zwischen der ersten und zweiten Phase. Diese gilt es zu begleiten, sodass ein selbstbestimmtes, gesundheitsförderliches und genussvolles Essverhalten sich entwickeln kann.

\section{Essen ist an Mahlzeiten gebunden}

In den verschiedenen Phasen lernen Kinder also, allmählich immer selbstständiger zu essen, genauer gesagt, sie lernen Mahlzeiten einzunehmen, denn: Gegessen wird im Kontext einer Mahlzeit, gemeinsam oder alleine. Die klassische Mahlzeit besteht aus mehreren Komponenten und wird am Tisch eingenommen. Das „Zwischendurch“-Essen, wie z. B. das Naschen oder - wie heute immer geläufiger - das „Snacken“, ist streng genommen eine Mahlzeit, denn hierfür wurden Lebensmittel beschafft und diese auch bewusst gegessen, wenn auch meist nicht - zumindest in der Retrospektive - bewusst verbucht. Bestimmte Mahlzeiten, wie das Frühstück, das Mittagessen und das Abendessen, ähneln sich in ihrer Struktur, in ihrem Aufbau. Routinen vom Einkauf über das Zubereiten bis hin zum Spülen können sich so einstellen und vereinfachen das Durchführen der Mahlzeiten. Diese Routinen zeigen sich besonders bei Festmahlzeiten, Weihnachts-, Geburtstagsessen, aber nicht nur dort in bestimmten Ritualen, die familiär/kulturell sehr verschieden sein können. Gelebte Rituale setzen Beziehung voraus und damit Kommunikation und eine gewisse Beständigkeit.

\section{RITUALE}

Mahlzeiten unterliegen einer Ordnung. Diese wird sozial und kulturell geprägt und wandelt sich ständig: Wie viele Mahlzeiten am Tag zu welcher Uhrzeit stattfinden und was zu den Mahlzeiten gereicht wird oder auch, ob sie warm oder kalt eingenommen werden.

Heute sind wir weg von dem Mahlzeiten-Tisch - hin zum Snack-Tisch. Dies hat nicht nur physiologische Konsequenzen, sondern auch familiäre, pädagogische und psychologische. So dienen gemeinsame Mahlzeiten auch als Vorbeugung von Heißhungerattacken, da sie rundum satt machen. Eine Familienmahlzeit ist Beziehungszeit, im besten Fall eine positive Anregungszeit. Nicht selten werden Mahlzeiten in Familien aber als Stresszeiten empfunden. So ermöglicht die moderne Snackkultur Familien, dem Stress am Tisch aus dem Weg zu gehen, zumindest vorerst.

Gemeinsame Mahlzeiten machen Familien stark. 


\section{Bedeutung einer Mahlzeit}

Wenn wir „Mahlzeit“ hören, denken wir zunächst daran, dass unser Bedürfnis nach Sättigung befriedigt wird. Aber eine Mahlzeit kann deutlich mehr! Sie erfüllt verschiedene menschliche Bedürfnisse. Im Vordergrund steht sicherlich die körperliche, physiologische Funktion. Hunger und Durst sollen gestillt und damit die Energie- und Nährstoffbilanz ausgeglichen werden. Eine Mahlzeit stiftet aber auch Gemeinschaft und bietet gute Möglichkeiten, Regeln und Normen zu lernen. Hier wird kommuniziert und Beziehungen werden gelebt, denn gemeinsam zu essen, ist Kommunikation.

Da wir alle essen müssen, ist eine Mahlzeit auf der zwischenmenschlichen Ebene das Ereignis, das uns am meisten und häufigsten verbindet. Gemeinsam zu essen, befriedet. Damit hat eine Mahlzeit auch eine soziale Funktion sowie eine psychische Funktion im Sinne von „Mahl-ZeitNehmen“. So werden die Bedürfnisse nach Sicherheit, Zugehörigkeit, Liebe, Wachstum und Wertschätzung befriedigt. Außerdem ist eine Mahlzeit nicht nur in Kita und Schule, sondern auch zu Hause automatisch eine pädagogische Bildungszeit. Nebenbei lernen Kinder - auch Erwachsene - Lebensmittel und deren Herkunft kennen, erfahren etwas über Zubereitungsarten. Es wird Esskultur vermittelt, bzw. es werden unterschiedliche Esskulturen kennengelernt. So ist eine Mahlzeit nicht nur ein Sättigungsort, sondern auch ein Kommunikationsort und Beziehungsort sowie ein Emotionsort, Erziehungsort und Bildungsort. Die soziale, psychische und pädagogische Funktion einer Mahlzeit sättigt den emotionalen Hunger, die physiologische Funktion den Hunger nach Kalorien und Nährstoffen. Der Mensch ist dann zufrieden mit einer Mahlzeit, wenn beide „Hunger“ gestillt werden. Mobiles Essen, häufig auch das Essen von Fast Food, führt auf der physiologischen Seite oft zu einer Überernährung und seelisch zu einer Unterernährung. Kalorisch satt, emotional hungrig. So kann es auch in Bewegung zu Übergewicht kommen.

Merke

Bei einer Mahlzeit sollten die „Tellerthemen“ (physiologische Sättigung) und die „Tischthemen“ (emotionale Sättigung) gleich relevant sein.

Bereits ein Säugling zeigt deutlich, wenn die Balance zwischen physiologischer und emotionaler Sättigung nicht gegeben ist. Wenn die Mutter beim Stillen ihre Aufmerksamkeit dem Smartphone widmet und nicht ihrem Kind, dann wird das Kind zwar über die Milch satt, bleibt aber emotional hungrig. Daraufhin meldet sich das Kind immer wieder, denn es ist emotional hungrig. Auch bei Jugendlichen kann man dies sehr gut beobachten, sie essen sehr gerne allein in ihrem Zimmer, essen die Pizza vor dem PC, sind danach kalorisch satt, fühlen sich aber noch hungrig und essen weiter. Sie versuchen so, ihren emotionalen Hunger mit Kalorien zu sättigen.

\section{Mahlzeiten strukturieren den Tag}

Kleine Kinder erfahren ihren Tagesablauf vor allem über die Mahlzeiten. Bestimmte Handlungen davor oder danach werden damit verbunden, geben Rhythmus und Sicherheit. Mahlzeiten machen so den Tag überschaubarer. Im Schul- und Berufsleben dienen Mahlzeiten oft dazu, dass der Tag eine Struktur bekommt und einem kleine Erholungsphasen sicher sind, aber auch Gespräche mit der Familie oder mit Freunden. Auch der Austausch mit Kollegen findet hier, beim gemeinsamen Essen, auf einer anderen als der rein beruflichen Ebene statt. Senioren bieten Mahlzeiten eine gefühlte Verkürzung des Tages, oft auch die einzigen Termine des Tages.

\section{Vereinbarungen bei Tisch}

Mahlzeiten geben nicht nur Struktur im Tagesablauf, sie unterliegen auch einem bestimmten Regelwerk. Beginnend mit der Sitzordnung, die jedes Familienmitglied seine Position in der Familie sicher fühlen lässt, über die bestimmte Reihenfolge der Speisen, bis zu dem Zeitpunkt, der die Mahlzeit beendet. Tischregeln, Tischmanieren sind zum einen kulturell festgelegt, können aber auch sehr familienspezifisch sein. Vonseiten der Erwachsenen verstehen wir meistens das Benehmen der Kinder bei Tisch, ja, was vielleicht den Umgang mit Serviette und Besteck betrifft! Es gibt aber auch Tischvereinbarungen für Eltern/ Erwachsene: Keine Konfliktgespräche, das Kind kann sich dem nicht entziehen, keine Kritik am Kind, das Kind nicht (sorgenvoll) beobachten, das Kind darf etwas nicht mögen, satt sein dürfen... und alles ist gut, nicht essen ist in Ordnung, Gespräche sind erwünscht, alle kommen zu Wort. Diese Tischregeln/-manieren sind sehr viel wichtiger als die für die Kinder und können Mahlzeiten zu Essenszeiten aufwerten, die Erholungspausen im Alltagsgeschehen bieten. Sie sind Strukturgeber, „Tankstellen“ für Energie und Emotionen, Genusszeiten, Sinnes- und heitere Zeiten. So hinterlässt das „Miteinander essen“ gemeinsame Familienmahlzeiten, die nachhaltige Erfahrung von Aufmerksamkeit, Geborgenheit, Sicherheit und zugetrauten Herausforderungen.

Merke

Gemeinsame Familienmahlzeiten ermöglichen Kindern körperliches und seelisches Wachsen.

\section{Einflussfaktoren auf das Essverhalten}

\section{Geschmacksentwicklung}

Bereits ab der 8. Schwangerschaftswoche bilden sich beim Fötus die ersten Geschmackszellen aus, ab der 12. Schwangerschaftswoche der Schluckreflex. So lernt der Fötus über das Trinken des Fruchtwassers (davon trinkt er bis zu 750 ml/Tag) und das Nabelschnurblut die Geschmacksstoffe der Familienernährung, die in diese Flüssigkeiten übergehen, kennen. Schmecken kann der Fötus „süß“, „sauer“ und „bitter“, wobei er auch hier schon „süß“ bevorzugt, „sauer“ und „bitter“ ablehnt. 


\section{Merke}

Die Geschmacksprägung beginnt bereits im Mutterleib.

Nach der Geburt prägt zunächst die Muttermilch den Geschmack auf vielfältige Weise, denn schon 1-2 Stunden nach dem Essen der Mutter befinden sich die Geschmacksstoffe des Essens in der Milch. Mit 6 Monaten können die Kinder dann „salzig“ schmecken, mit 9 Monaten kommt „umami“ hinzu. Umami steht für „eiweißreich“, „fleischig“ und ein wenig „fettig“. Die Entwicklung der Geschmacksknospen ist mit 3 Jahren physiologisch abgeschlossen, das Kind kann alles schmecken - die Entwicklung des Geschmackssinns geht aber ein Leben lang weiter.

Kinder kommen mit rund 10000 Geschmacksknospen auf der Zunge und im Mundraum auf die Welt. Sie sind wahre Geschmackskünstler und können so gut riechen und schmecken, wie wir Erwachsene es uns nicht vorstellen können. Denn Kinder kommen sozusagen mit sehr langen „Sinnesantennen“ auf die Welt, damit sie die Welt mit allen Sinnen in sich aufnehmen können. Im Erwachsenenalter degenerieren die Sinne nach und nach. Erwachsene haben nur noch halb so viele Geschmacksknospen wie die Säuglinge.

\section{PRAXISTIPP}

Eine ausgewogene, abwechslungsreiche vollwertige Ernährung während der Schwangerschaft und Stillzeit und der Zeit der Beikost hat einen enorm positiven Einfluss auf die Geschmacksprägung des Kindes. Die Vorbildfunktion beginnt sehr früh!

\section{Die biologischen Sicherheits- und Schutzprogramme}

Evolutionsgeschichtlich/genetisch fest verankert in jeder unserer Zellen wirken die biologischen Sicherheits- und Schutzprogramme. Sie haben sich im Laufe der Entwicklung des Menschen ausgebildet, um den Körper vor Gefahren zu schützen und dafür zu sorgen, dass der Organismus mit den notwendigen Nährstoffen versorgt wird. Diese Programme hatten zu Zeiten, als der Mensch noch als Jäger und Sammler unterwegs war, einen Sinn. Sie haben u. a. für das Überleben der Menschheit gesorgt. Heute, im 21. Jahrhundert, sind diese Programme nicht mehr notwendig, wir brauchen sie nicht für unser Überleben, denn wir haben sichere Lebensmittel, die uns gut versorgen, vor Vergiftungen, Verkeimungen und Mangel schützen und durchweg rund um die Uhr verfügbar sind. Hinzu kommt, dass diese Programme der Entwicklung eines gesundheitsförderlichen Essverhaltens, das heute bei ständiger Lebensmittelverfügbarkeit und mangelnder Bewegung notwendig ist, entgegenwirken.

Die biologisch-evolutiven Programme zeigen sich bei Kindern sehr deutlich und haben einen enormen Einfluss auf ihre Lebensmittelauswahl, ihr Essverhalten, da Kinder intuitive Esser sind. Erwachsene haben das Essen erlernt und reflektieren das Essen, sind somit kognitive Esser.

\section{Steuerprogramme}

Ich liebe Süßes ... denn reife, süße Früchte und Gemüse sind reich an dem schnellen Energielieferanten Zucker sowie reich an Vitaminen und Mineralstoffen.

Ich lehne Bitteres und Saures ab ... denn bittere und saure Früchte und Gemüse geben eher einen Hinweis auf Unreife, Verdorbenheit und potenzielle Giftigkeit.

Ich liebe Salziges ... denn der Körper braucht kleine Mengen Salz für seinen Organismus. Früher war Salz sehr schwer zu beschaffen. So sorgte das Bedürfnis nach salzigem Geschmack dafür, dass unsere Vorfahren nach Salz suchten, um überleben zu können.

Ich liebe konzentrierte Energie ... denn Lebensmittel mit einem hohen Energiegehalt haben viel Fett und machen lange satt.

Früher war eine zeitnahe Mahlzeit nicht gesichert, diese konnte auch einmal ein paar Stunden oder Tage auf sich warten lassen. So hatte es keinen Sinn, Lebensmittel mit einem großen Volumen und wenig Energie zu bevorzugen, denn diese füllen den Magen nur kurzfristig und machen nicht lange satt. Die Suche nach konzentrierter Energie ist insbesondere bei kleinen Kindern sehr stark ausgeprägt. Denn sie haben einen sehr kleinen Magen verbunden mit einem sehr hohen Energiebedarf. Übrigens: Muttermilch erfüllt diese genetisch bedingten Vorlieben perfekt, sie ist süß und fett, enthält kleine Mengen Salz und ist nur dann bitter, wenn die stillende Frau eine große Menge bitterer Lebensmittel gegessen hat, z.B. Spargel, was nicht selten dazu führt, dass die Milch dann abgelehnt wird.

Diese 4 evolutiven Programme einmal zusammengefasst zeigen, dass wir Menschen eine Vorliebe für süße, salzige und fette Lebensmittel haben und bittere bzw. saure Lebensmittel ablehnen. Da passt ein noch so dringend empfohlenes und zu einer wunderschönen Landschaft dekoriertes Brokkoliröschen überhaupt nicht hinein. Dieses Brokkoliröschen gibt dem Kind nicht die Information, von ihm lange satt und versorgt zu werden. Das bedeutet, dass der kritische Blick der Kinder auf das Gemüse kein Trotz gegen den Eltern- bzw. Erzieherinnen-Willen ist, sondern eine pure Überlebensstrategie.

\section{Umsetzung in die Praxis}

Wenn Erwachsene also „wollen“, dass Kinder Gemüse essen „sollen“, beziehungsweise, wenn Kinder das essen wollen, was sie sollen, dann sollte das gegarte Gemüse immer mit konzentrierter Energie angeboten werden. Mit Vollkornprodukten, Hülsenfrüchten, Fleisch, Fisch oder Eiern. Dann bekommen die Kinder das richtige Signal: Hier werde ich satt! 
Frisch aufgeschnittenes Gemüse, wie Möhren, Kohlrabi, Gurke und Paprika essen viele Kinder sehr gerne, insbesondere, wenn sie es dippen können. Denn hier haben sie nicht den Anspruch, satt werden zu wollen. Bei Frischkost geht es um Spaß, Krachendes, Buntes und Saftiges.

\section{Merke}

Die Geschmacksrichtungen „süß“ und „fett“ sind nicht nur genetisch positiv belegt, sondern auch über Er-

fahrungen und unsere Esskultur.

Die Kinder bekommen gleich zum Start ins Leben monatelang allersüßeste und fette Muttermilch in allerengster Liebesbeziehung und engem Körperkontakt und machen die Erfahrung, satt, rund und groß zu werden, besser geht es nicht. Schauen wir auf unsere Esskultur: Immer dann, wenn es uns schon so richtig gut geht, an Weihnachten, Ostern, am Geburtstag, wird dieses Gefühl mit etwas Süßem und Fetten noch unterstützt. Weihnachtskekse, Schokoladeneier und Geburtstagskuchen erfüllen genau diese Funktion, und im Sommer, wenn das Wetter schön ist, toppen wir unser ohnehin schon gutes Gefühl mit einem Eis. So ist Süßes und Fettes immer mit liebevoller Zuwendung und guter Atmosphäre verbunden, was die genetisch angelegte Vorliebe unterstützt. Bitteres wird eher mit negativen Situationen verbunden, mit Druck bis hin zur Erpressung, mit Ablehnung und unter Umständen auch Ekel - „Nur wer Rosenkohl isst, bekommt auch Nachtisch“.

\section{PRAXISTIPP}

Idealerweise bietet man Lebensmittel bzw. Gerichte, die aufgrund der biologischen Programme eher abgelehnt werden, in guter und angenehmer Atmosphäre an - also ohne Druck, möglichst selbstverständlich, „in Beziehung“, und kombiniert diese eher bitteren Lebensmittel mit vertrauten, geliebten Komponenten und möglicherweise auch mit süßen und fetten, sattmachenden Ergänzungen.

\section{Steuerprogramme treffen auf}

\section{Entwicklungsaufgaben}

Ich verlasse mich auf meine Eltern ... denn, bis die Kinder ungefähr 2 Jahre alt sind, fühlen sie sich noch nicht in der Lage, die Lebensmittel nach ihrer Sicherheit und dem Überlebenswert selbst zu beurteilen. Daher essen sie am liebsten das, was ihre Eltern auch essen und noch lieber von einem gemeinsamen Teller. Und richtig gut wird es dann, wenn die Kinder auf ihrem Schoß bzw. auf dem eines vertrauten Menschen sitzen dürfen, dann fühlen sie sich rundum sicher. Nicht selten nehmen die Kinder auch ein wenig Essen, z. B. ein Stückchen Gemüse von dem Teller und bieten es dem Schoßgeber an ( $\mathbf{A} \mathbf{b} \mathbf{b} \mathbf{b} \mathbf{2}$ ). Das ist ein Zeichen von tiefem Vertrauen, das Kind überprüft mit

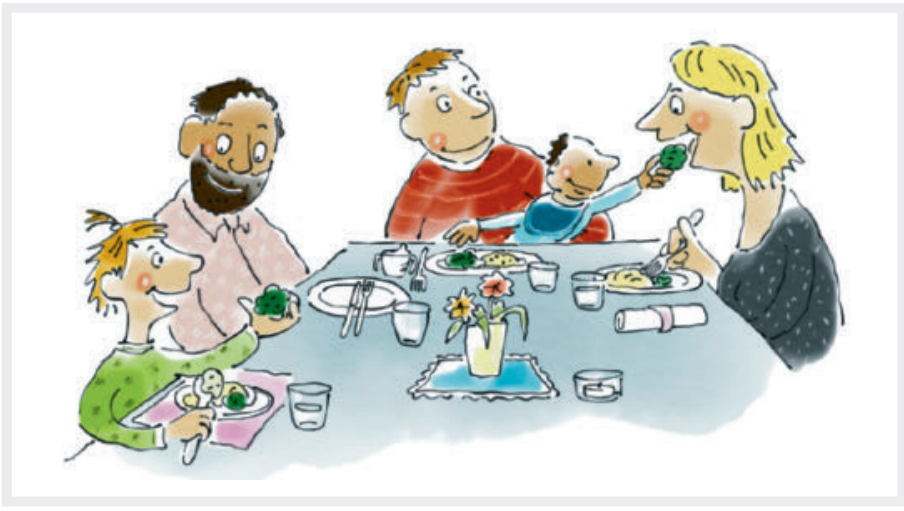

Abb. 2 Ich verlasse mich auf meine Eltern. Quelle: Verlag Eugen Ulmer, Stuttgart, mit freundlicher Genehmigung

dem Anbieten die Sicherheit und den Lebenswert dieses Stückchen Gemüses.

\section{Umsetzung in die Praxis}

Das Angebot des Kindes sollte angenommen werden. Nur so kann es lernen zu vertrauen. Das Kind sieht: Meine Mutter/mein Vater isst das Stückchen Gemüse und erfährt darüber, dass dieses Essensangebot sicher ist. Dem gleichen Angebot auf dem eigenen Teller traut es deswegen noch nicht. So ist das Essen von einem Teller sehr vorteilhaft. Das Kind fühlt sich sicher in seinem Tun und die Eltern können sich darüber freuen, dass ihr Essensangebot angenommen wird und ihr Kind lernt, dem Essen zu vertrauen und sich wieder ein Stückchen mehr daran zu gewöhnen, sein Geschmacksgedächtnis zu erweitern.

Ich habe Angst vor Unbekanntem (Neophobie) ... denn dies ist ein angeborenes Sicherheitsprinzip, das zwischen $1 \frac{1}{2}$ und $2 \frac{1}{2} 2$ Jahren am stärksten wirkt. Dies hat die Natur klug eingerichtet, denn zu diesem Zeitpunkt sind die Kinder bereits mobil und könnten sich alles Mögliche bereits in den Mund stecken. So schützt die Angst vor Unbekanntem vor Gesundheitsschädlichem, das z. B. irgendwo auf dem Boden liegt. Wenn ein Lebensmittel anders aussieht, riecht oder schmeckt als gewohnt, lehnt ein Kind es in den ersten Lebensjahren häufig ab.

Nicht alle Kinder sind in ihrer Lebensmittelauswahl vorsichtig. Es gibt Kinder, und diese Kinder hat es immer schon gegeben, die das Essen und die Welt der Lebensmittel als ihr „Hobby“ auserkoren haben. Sie haben eine vielleicht angeborene Leidenschaft für das Essen. Diese „Hobbyesser“ lassen sich von genetischen Programmen nicht irritieren, machen stets gute Erfahrungen, und wenn sie eine unangenehme Erfahrung machen, ist diese nicht nachhaltig.

Merke

Dem „Hobbyesser“ steht der „Supertaster“ gegenüber.

Einige Kinder kommen mit der doppelten Anzahl Geschmacksknospen auf die Welt, mit 20000 , und sind hoch- 


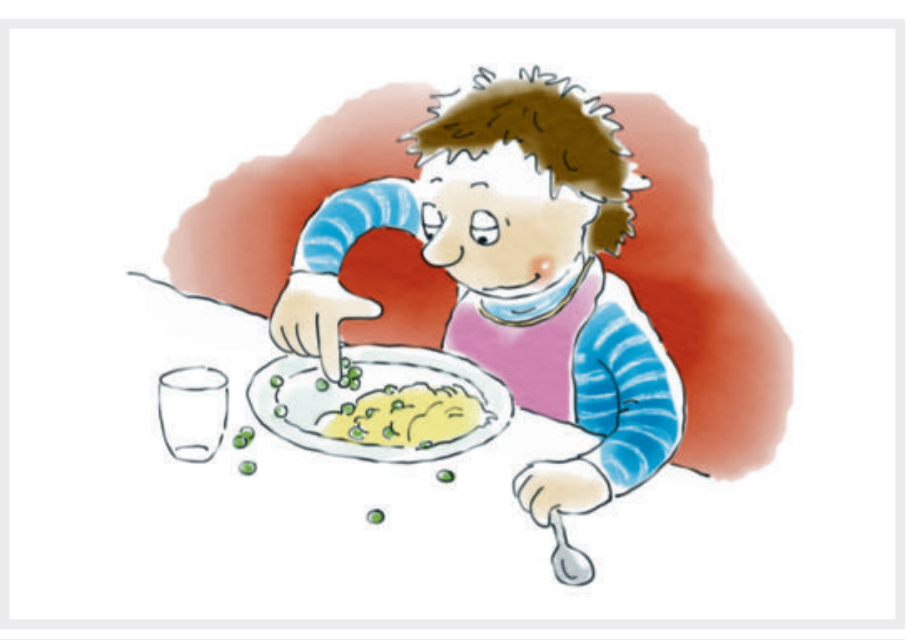

$\triangle$ Abb. 3 Ich verlasse mich auf mich. Quelle: Verlag Eugen Ulmer, Stuttgart, mit freundlicher Genehmigung

sensibel in Bezug auf Geschmack, Geruch und Konsistenz im Mundraum. Für Erwachsene nicht vorstellbar. Der Supertaster schmeckt aus Gerichten einzelne Komponenten heraus und so können kleinste Veränderungen in einer bekannten Speise dazu führen, dass sie ihnen direkt auffallen und dies zu einer schlechten Erfahrung führt. So begleitet sie die Angst vor Unbekanntem länger. Auch Konsistenzen stellen Herausforderungen dar - z. B. frische Tomaten mit ihrer glatten Haut, dem mürben Fruchtfleisch und den „glibberigen“ Samen sind für Supertaster eine unglaubliche Herausforderung, püriert in der Sauce aber kein Problem.

\section{Umsetzung in die Praxis}

Lieblingsgerichte sollten bei Supertastern nicht verändert werden und immer gleich zubereitet und angeboten werden. So kann das Kind immer gute Erfahrungen machen. Wenn das Brot nicht wie gewohnt in 4 Quadrate geschnitten ist, sondern in 2 Dreiecke, könnte das so irritieren, dass das Brot gar nicht erst angerührt wird. Wenn das Kind dann doch einmal etwas Unbekanntes in den Mund genommen hat und erst dann merkt, dass es dieses Lebensmittel nicht kennt, kann es sein, dass es dieses ausspuckt. Das lässt man am besten einfach zu, und wenn das Kind dies in den Backentaschen hortet, animiert man es, dies auszuspucken.

Ich versuche, mich auf mich selbst zu verlassen ... denn ab dem 2. Geburtstag entwickelt sich langsam das Autonomiebestreben der Kinder, sie möchten zunehmend die Dinge selbst entscheiden und beurteilen sowie lernen, sich selbst zu vertrauen. So auch beim Essen. Das bedeutet, dass das Kind sich nicht mehr durchgängig auf die Erwachsenen verlässt, sondern es versucht selber abzuwägen - das geschieht schneller oder langsamer, bewusster oder unbewusster, klammheimlich oder laut tönend - sicherlich nicht immer gleich, also kindgemäß. Das Kind versucht, die Lebensmittel selbst nach ihrem Überlebenswert und ihrer Sicherheit einzustufen und dies gelingt an einem
Tag sicherer und besser, am nächsten unsicherer und skeptischer. Also was gestern noch superlecker war, kann heute schon vehement abgelehnt werden.

Kinder lieben in diesem Alter - bis ca. 6 Jahre - Übersicht auf dem Teller. Sie möchten klar erkennen können, was sich in den Schüsseln befindet, bevor sie sich nehmen. Und nicht selten essen sie die verschiedenen Komponenten nacheinander, also getrennt und so pur wie möglich ( $>$ Abb. $\mathbf{3}$ ). Nur so können sie herausfinden, ob diese Lebensmittel sicher sind. Aus der Perspektive eines Erwachsenen erscheint das eher unattraktiv, wenn die Kinder die Nudeln pur essen, um im Anschluss die Sauce mit Hingabe zu löffeln.

\section{Umsetzung in die Praxis}

Das nicht immer konsequente Autonomiebestreben von Kindern auch beim Essen sollte respektiert und nicht bewertet werden.

Wenn gestern der Grünkohl sehr lecker war und heute absolut ungenießbar ist, dann bleibt diese Situation möglichst unkommentiert. Das Kind sichert sich selbst ab, denn heute vermittelt der Grünkohl ihm nicht so sichere Signale. Ein Satz wie: „Aber gestern mochtest Du den Grünkohl doch so gerne“, zeigt dem Kind nur, dass ihm heute etwas, also das Grünkohlessen, nicht gelingt und nimmt ihm die Motivation, sich in der Welt der Lebensmittel zurechtfinden zu wollen.

Ich mag plötzlich mein Lieblingsessen nicht mehr (spezifisch sensorische Sättigung) ... denn dieses Programm sorgt dafür, dass das Kind nicht zu einseitig isst und daraufhin womöglich einen Mangel an bestimmten Nährstoffen bekommt. Es kann passieren, dass es 6 Monate lang das gleiche Müsli mit dem gleichen Obst isst - das wird auch als sehr lecker und gut empfunden - und dann von heute auf morgen beschließt, es nicht mehr zu essen, es nicht zu mögen und auch mit Überzeugung zu behaupten, es noch nie gemocht zu haben.

Merke

Die spezifisch sensorische Sättigung ist der Auslöser dafür, dass wir Abwechslung in unsere Essenswelt bringen.

\section{Umsetzung in die Praxis}

Das, was ein Kind ein halbes Jahr gerne gegessen hat und es jetzt plötzlich mit der Aussage „Das mag ich nicht“ ablehnt, sollten wir als Erwachsene übersetzen. Das heißt nichts anderes als „Mein Körper braucht Neues“. Dies können die Kinder aber so nicht ausdrücken, also auch hier: Nicht kommentieren, sondern gemeinsam etwas Neues suchen.

\section{Beziehung}

Essen ist von Anfang an in zwischenmenschliche Interaktionen fest eingebunden. Die alte Volksweisheit „Essen 
und Trinken hält Leib und Seele zusammen “ beschreibt, dass Essen und Trinken für unsere Seele genauso wichtig sind wie für unsere körperlichen Vorgänge - so hat Essen immer auch etwas mit Beziehung zu tun.

Kinder lernen Essen in der Auseinandersetzung mit ihrer Umwelt, den körperlichen, seelischen und sozialen Beziehungssystemen. Wie Kinder das Essen lernen, wird somit von verschiedenen Beziehungen beeinflusst. Von den Beziehungen zu den Menschen, von denen sie das Essen lernen und gelernt haben (Eltern, Großeltern, Geschwister, Tageseltern und Erzieher), und den Menschen, mit denen sie in der aktuellen Situation essen, z. B. zu Hause, in der Kita, in der Schule. Essen hat aber auch mit der Beziehung des Kindes zu sich selbst zu tun, zu seiner momentanen Stimmung und dem allgemeinen Selbstwertgefühl. Zu guter Letzt hat Essen auch mit der Beziehung zu den Lebensmitteln selbst zu tun, welche Erfahrungen mit einzelnen Lebensmitteln gemacht worden sind. Die Linsensuppe bei den Großeltern, die gemeinsam mit Zeit und Ruhe gekocht worden ist, die die Lieblingssuppe des Großvaters ist, die nur als „Angebot“ auf dem Tisch steht, da die Großeltern keine Sorge haben, wenn das Kind nicht isst (sie haben ja keinen Erziehungsauftrag), mit ihr wird das Kind vermutlich sehr gute Erfahrungen machen, sodass die Linsensuppe positiv abgespeichert wird.

\section{PRAXISTIPP}

Damit Kinder eine gute Beziehung zum Essen aufbauen können, brauchen sie Erwachsene, zu denen sie eine gute Beziehung haben und die selbst eine gute Beziehung zum Essen haben.

Eine Beziehung fühlt sich gut an, wenn die Balance zwischen Autonomie und Verbundenheit gegeben ist. So haben alle Erwachsenen, Eltern, Großeltern, Erzieher und Lehrer, die die Kinder beim Essen begleiten, den Auftrag, ihre Beziehung zu dem Kind zu überprüfen, wie viel Autonomie sie dem Kind beim Essen zusprechen und die eigene Beziehung zum Essen zu reflektieren.

\section{Umsetzung in die Praxis}

Alle Erwachsenen, die Kinder beim Essen begleiten, sollten ihr Essverhalten, ihre Beziehung zum Essen und ihre eigene Essbiografie reflektieren. Wie viel Autonomie kann man dem Kind einräumen, eigene Entscheidungen zu treffen, die von den Erwachsenen-Ideen und -Überzeugungen abweichen, und welchen Einfluss hat dies auf die Beziehung zwischen dem Kind und dem Erwachsenen.

Folgende Fragen sollten sich die Erwachsenen stellen:

- Wie wichtig ist es mir, dass mein Kind eine gute Beziehung zum Essen aufbaut?

- Wie gut gelingt es mir, meinem Kind in Essensangelegenheiten zu vertrauen?
- Wie wichtig ist es mir, dass mein Kind auch außer Haus beim Essen und mit dem Essen gute Erfahrungen macht?

- Wie wichtig ist es mir, dass mein Kind außer Haus zur Essensgemeinschaft gehört?

\section{Erfahrungen}

Es sind die positiven Gefühle (Erfahrungen), die zur Annahme führen. Denn alles, was wir mit positiven Gefühlen tun, da fühlen wir uns hingezogen, da sind wir konzentriert und motiviert und da entstehen auch wieder positive Gefühle. Wenn Kinder - und bei Erwachsenen ist es nicht viel anders - beim Riechen oder Schmecken eines Lebensmittels - insbesondere, wenn sie dazu gedrängt werden - negative Gefühle empfinden, sei es, dass da ein Lebensmittel zu bitter ist oder möglicherweise auch mit Ekel verbunden wird, dann wollen sie diese Lebensmittel erst einmal nicht essen, je nachdem wird dieses Lebensmittel auch über Jahre vehement abgelehnt. Es ist sogar so, dass, wenn nach dem Genuss eines geliebten Lebensmittels sich kurz danach eine Magen-Darm-Grippe ansagt und das Essen erbrochen wird, dieses Lieblingsessen erst einmal nicht angerührt wird. Denn die negative Erfahrung ist deutlich stärker als die vorherigen positiven Erfahrungen.

\section{Umsetzung in die Praxis}

Damit Kinder positive Erfahrungen mit dem Essen machen können, brauchen sie einen „Lebens-Ess-Raum“, der ihnen dieses ermöglicht. Dazu gehört in erster Linie die Erlaubnis, die Lebensmittel mit allen Sinnen erfahren zu dürfen. Kinder lernen über die Sinne, nicht über den Verstand. Und so ist es wichtig, diese Erkundungen auch am Esstisch zu ermöglichen. Hier geht es nicht um das Spielen mit Lebensmitteln. Kinder brauchen bis zu 20 Kontakte mit ein und demselben Lebensmittel („Mere-ExposureEffekt“), und diese Kontakte sind keine (!) Geschmackskontakte, sondern Sicht-, Hör-, Fühl-, Riechkontakte - erst zum Schluss kommen die Geschmackskontakte. Man gibt dem Kind die Möglichkeit, mit den Lebensmitteln zu flirten, ein Flirt ist ganz unverbindlich und geht nur über den Blick. Und ob aus einem Flirt eine Verabredung, ein Kennenlernen, ein Liebenlernen wird oder eine lebenslange Liebesbeziehung, das weiß man doch noch nicht. Nicht selten werden Kinder ermutigt, vor dem Flirten zu küssen, das ist übergriffig.

\section{Merke \\ Es gilt: Kein Kind muss probieren!}

Im Jugend- und Erwachsenenalter ist das anders. Dort gilt: Wir mögen die Dinge, weil wir sie so oft gegessen haben. Diese anfängliche Motivation, etwas zu essen, was noch nicht richtig gut schmeckt, fehlt den kleinen Kindern, aber durch die Begleitung von freundlichen Erwachsenen können Kinder positive Erfahrungen machen und sind so motiviert. 


\section{Erziehung}

Mahlzeiten sollten angepasst gestaltet werden an die Bedürfnisse der Kinder, deren Alter und auch Entwicklung, z. B. in Bezug auf die Verdauungsorgane, Zahn- und motorische Entwicklung und an ihre momentane Verfassungalso pädagogisch begleitet sein. Erwachsene sollten sich an dem „Wollen“ der Kinder orientieren, das eigene und vielleicht das gesamtgesellschaftliche „Sollen“ im Blick haben, es nicht verlieren und gegebenenfalls eine gezielte Soll-Anpassung vornehmen und dabei die Grundideen der Esserziehung bewusst machen.

- Wir haben Spaß am Essen.

- Wir bestimmen, wann (wie oft), was und wie es etwas zu essen gibt - also das Gesamtsetting „Mahlzeit“, und die Kinder entscheiden, ob und wie viel sie essen möchten und darin vertrauen wir ihnen. Für beide Parteien gilt: Ein Nein ist ein Nein!

- Wir animieren zum Flirten, akzeptieren die Ablehnung und unterstützen die Freiwilligkeit.

- Wir sind authentisch, unser Sollen und Wollen stimmen überein.

- Wir unterstützen das Essen mit allen Sinnen.

- Wir geben Zeit und Raum für Entwicklung.

- Wir versüßen das Leben und nicht das Essen - Essen wird weder als Belohnung noch zur Bestrafung eingesetzt.

- Wir stellen gemeinsam Regeln auf und halten uns an diese.

Wie die Vereinbarungen mit den Kindern besprochen und wie ausführlich ihre Hintergründe erklärt werden sollten, hängt von dem Alter der Kinder ab. Je jünger die Kinder sind, desto eher ist es angemessen, die Vereinbarungen erst einmal selbst aufzustellen und vorzuleben. Gegebenenfalls kann man situativ erklären und später dann gemeinsam aufstellen, verändern und anpassen an das Familienleben.

\section{Umsetzung in die Praxis}

Gemeinsame Vereinbarungen könnten so aussehen:

- Alle helfen mit, jeder so gut, wie er kann (Einkauf, Zubereitung, Tisch decken und abdecken).

- Jede Mahlzeit hat einen Anfang und ein Ende und zwischen 2 Mahlzeiten wird mindestens 2 1 12 Stunden nichts gegessen.

- Wir beginnen und beenden die Mahlzeit gemeinsam.

- Jeder nimmt sich selbst von dem, was er sich nehmen möchte und so viel, wie er möchte mit dem Blick auf die gemeinschaftliche/familiäre Verteilung.

- Wer etwas nicht essen möchte, sagt: „Das möchte ich nicht essen.“

\section{KERNAUSSAGEN}

- Essen ist von Anfang an in zwischenmenschliche Interaktionen fest eingebunden.

- Die Entwicklung des Essverhaltens vom Säugling bis ins Erwachsenenalter lässt sich in 6 Phasen einteilen.

- Diese Phasen sind geprägt von der Entwicklung des Verdauungstraktes, der Motorik sowie der physischen und psychischen Bedürfnisse.

- Gemeinsame Mahlzeiten stillen dabei nicht nur den physischen, sondern auch den emotionalen Hunger der Kinder.

- Biologische Sicherheits- und Schutzprogramme steuern, welche Nahrungsmittel Kinder bevorzugen und (zunächst) ablehnen.

- Erst durch positive Erfahrung und vermittelt durch Vertrauenspersonen erweitert sich das Nahrungsmittelspektrum im Laufe der Zeit.

- Im Spannungsfeld „Bindung - Ablösung - Autonomie“ lernt das Kind verschiedene gesellschaftliche und kulturelle Regeln rund um die Einnahme von Mahlzeiten kennen.

- Dabei sollten alle Erwachsenen, die Kinder beim Essen begleiten, ihr Essverhalten, ihre Beziehung zum Essen und ihre eigene Essbiografie reflektieren.

\section{BUCHTIPP}

Edith Gätjen, Markus Keller

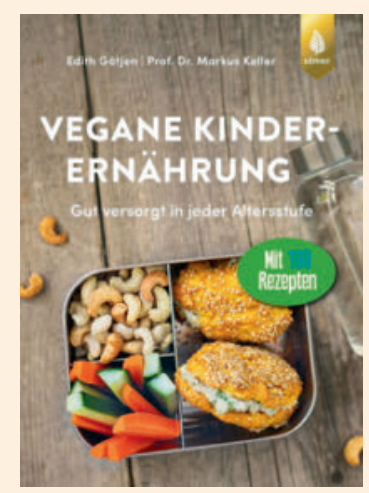

Vegane Kinderernährung. Gut versorgt in jeder Altersstufe. Mit über 100 Rezepten. Verlag Eugen Ulmer; 2020. 256S., 79 Farbfotos, 56 farbige Zeichnungen, 15 Tabellen. Gedruckt: ISBN 978-38186-0959-7. € 28,00. E-Book (pdf): € 18,99. Der Ratgeber gibt Antworten auf alle Fragen rund um die vegane Ernährung von Klein- und Grundschulkindern auf Basis aktueller wissenschaftlicher Erkenntnisse für vegan lebende Familien, Ernährungsfachkräfte und Kinderärzte. 
Interessenkonflikt

Die Autorin gibt an, dass kein Interessenkonflikt besteht.

Autorin

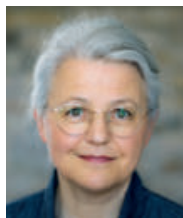

\section{Edith Gätjen}

Edith Gätjen ist Ökotrophologin, Systemische Paar- und Familientherapeutin und Supervisorin, psychologische Beraterin, Lehrbeauftragte an der Hochschule für Gesundheit in Bochum und in verschiedenen Aus- und Weiterbildungsbereichen als Ausbildungsleiterin tätig, u. a. für den UGB und die Sarah Wiener Stiftung. Sie arbeitet in eigener Praxis für systemische Essund Familientherapie. Ihr Hauptschwerpunkt liegt in der vegetarischen und veganen Familienernährung. Dazu hat sie auch Fachbücher veröffentlicht. Foto: Sarah Wiener Stiftung/ Thomas Koehler/photothek.net

\section{Korrespondenzadresse}

\section{Edith Gätjen}

Hofgut Mielenforst

Mielenforster Kirchweg 56

51069 Köln

edith@gaetjen.de

Bibliografie

DOI https://doi.org/10.1055/a-1115-9227

Ernährung \& Medizin 2020; 35: 81-89

(c) Georg Thieme Verlag KG Stuttgart · New York

ISSN 1439-1635 